\title{
Improved local spectrum sensing for cognitive radio networks
}

\author{
Waleed Ejaz ${ }^{1}$, Najam ul Hasan', Muhammad Awais Azam² and Hyung Seok Kim
}

\begin{abstract}
The successful deployment of dynamic spectrum access requires cognitive radio (CR) to more accurately find the unoccupied portion of the spectrum. An accurate spectrum sensing technique can reduce the probability of false alarms and misdetection. Cooperative spectrum sensing is usually employed to achieve accuracy and improve reliability, but at the cost of cooperation overhead among CR users. This overhead can be reduced by improving local spectrum sensing accuracy. Several signal processing techniques for transmitter detection have been proposed in the literature but more sophisticated approaches are needed to enhance sensing efficiency. This article proposes a two-stage local spectrum sensing approach. In the first stage, each CR performs existing spectrum sensing techniques, i.e., energy detection, matched filter detection, and cyclostationary detection. In the second stage, the output from each technique is combined using fuzzy logic in order to deduce the presence or absence of a primary transmitter. Simulation results verify that our proposed technique outperforms existing local spectrum sensing techniques. The proposed approach shows significant improvement in sensing accuracy by exhibiting a higher probability of detection and low false alarms. The mean detection time of the proposed scheme is equivalent to that of cyclostationary detection.
\end{abstract}

Keywords: Cognitive radio, Spectrum sensing, Primary user, Secondary user, Software-defined radio

\section{Introduction}

Wireless networks are regulated today by using a static spectrum allocation policy. However, an outsized portion of the spectrum is used sporadically and the utilization of the assigned spectrum ranges from 15 to $85 \%$, as illustrated in Figure 1 [1]. The growing number of wireless technologies and new applications are considerably increasing the demand for more bandwidth. Such stringent requirements cannot be met with the conventional inflexible spectrum management approaches in which each operator is granted an exclusive license to operate. As most of the useful radio spectrum has already been assigned, vacant spaces are difficult to find for setting up new services or add to existing services [2].

Cognitive radio (CR) is renowned for significantly enhancing the efficient utilization of the radio electromagnetic spectrum, which is considered a precious natural resource. $\mathrm{CR}$ is an intelligent wireless communication

\footnotetext{
* Correspondence: hyungkim@sejong.ac.kr

'Department of Information and Communication Engineering, Sejong University, Seoul, Republic of Korea

Full list of author information is available at the end of the article
}

system built on the top of software-defined radio (SDR) that learns from experience. By making use of both the intelligence and re-configurability, the radio can switch across the spectrum adaptively. Reconfiguration is performed by SDR, while CR relies on signal processing techniques for intelligence [2]. CR focuses on

- Efficient spectrum utilization

- Seamless communication of both CR users and licensed users

Only an unallocated portion of the spectrum or white space can be utilized by a secondary user (SU, i.e., unlicensed users using $\mathrm{CR}$ ). Therefore, a $\mathrm{SU}$ searches through the available spectrum for white space [3,4], a process called spectrum sensing. The prime concerns of spectrum sensing are that primary users (PU, i.e., licensed users) should not be disturbed by SU communication and that spectrum holes should be detected efficiently for maintaining the required throughput and quality of service.

\section{空}

(C) 2012 Ejaz et al.; licensee Springer. This is an Open Access article distributed under the terms of the Creative Commons Attribution License (http://creativecommons.org/licenses/by/2.0), which permits unrestricted use, distribution, and reproduction in any medium, provided the original work is properly cited. 


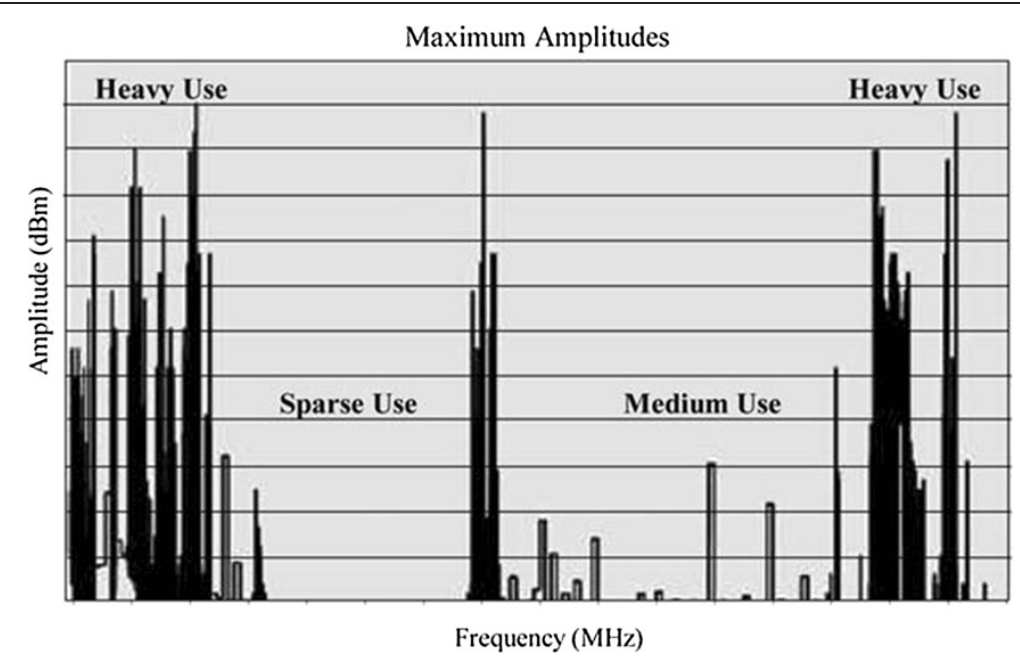

Figure 1 Fixed spectrum utilization [1].

The most important local sensing techniques considered for CR are matched filter detection, energy detection, and cyclostationary detection [5]. Energy detection needs much less sensing time but performs poorly under low signal-to-noise ratio (SNR) conditions. One of the well-known coherent detection techniques in the field for spectrum sensing is matched filter detection. Cyclostationary detection provides reliable detection but is computationally complex.

Metrics for detection performance are the probability of detection and false alarms. The probability that a SU declares that a PU is present when the spectrum is idle is called the probability of a false alarm. Conversely, the probability that the SU declares that the PU is present when the spectrum is occupied by the PU is called the probability of detection. The probability of misdetection indicates the probability that the SU declares that the $\mathrm{PU}$ is absent when the spectrum is occupied. CR should exhibit a low probability of false alarm and a high probability of detection. Misdetection leads to interference with the PUs, while false alarms decrease the efficiency of spectrum utilization [6].

Fuzzy logic has been proposed to solve many telecommunication problems since the 1990s. Applications of fuzzy logic to CR systems are discussed in [7]. Fuzzy logicbased cooperative spectrum sensing is proposed in [8] in which estimated results of SUs are combined to get a final result at the fusion center. In this article, we propose a two-stage fuzzy logic-based detection (FLD) system for local spectrum sensing. In the first stage, each CR performs existing spectrum sensing techniques, i.e., energy detection, matched filter detection, and cyclostationary detection. In the second stage, the outputs of those detection approaches are combined using fuzzy logic in order to deduce the presence or absence of primary transmitters.
The remainder of the article is organized as follows. Section 2 highlights the related work on transmitter detection techniques for spectrum sensing. The system model is presented in Section 3. The proposed fuzzy logic-based spectrum sensing approach is discussed in Section 4. Section 5 presents the numerical results confirming the accuracy of the simulation results and comparisons of the proposed approach with other detection techniques. Finally, conclusions are in Section 6.

\section{Related study}

Spectrum sensing plays a critical role for the efficient utilization of the radio spectrum. Researchers currently focus on two major aspects in spectrum sensing: (1) how to improve local sensing results and (2) cooperative spectrum sensing for better data fusion results.

Cooperative spectrum sensing is a two-stage process composed of (1) local sensing and (2) fusion of local sensing results. In the first stage, each SU sniffs the spectrum and deduces the presence or absence of PU. In the second stage, local decisions of multiple users are fused together for making the final decision on whether a PU is absent or present. For improving cooperative sensing, researchers focus on how to optimally fuse local sensing results. Several optimal fusion schemes for cooperative spectrum sensing have been summarized in [6]. Although fusion rules may improve the final decision, the decision is highly dependent on the result of the first stage. Therefore, improving the first stage can improve cooperation results.

Researchers have recently focused on how to achieve reliable results with less mean sensing time. The most promising reforms applied to local spectrum sensing are: using multiple antennas, using two-stage sensing 
schemes, and improving existing techniques. In $[9,10]$, the improvement of the sensing performance of energy detection is achieved using multiple antennas at the sensing node. In [11-14], two-stage spectrum sensing techniques are explored, in which the first stage involves coarse sensing and the second one involves fine sensing. In the majority of two-stage sensing techniques, coarse sensing performs energy detection while fine sensing is later performed to verify the presence or absence of PUs.

To improve the existing techniques, one-order cyclostationary detection in the time domain is proposed in [15], where the mean characteristic of the PU signal is exploited in order to improve the efficiency of channel sensing. Both real-time operation and low computational complexity can be achieved using this detection scheme. In [16], the energy detection technique is improved by replacing the squaring operation with arbitrary positive power operation. Power operation depends on the probability of false alarms, the probability of detection, the average SNR, and the sample size. By choosing the value of the power operation, detection performance of a conventional energy detector can be improved. Advanced sensing techniques for energy detection, including multiple antenna sensing and cooperative sensing, are discussed in [17].

L. A. Zadeh first introduced fuzzy logic in order to cover more general linguistic notation for extending binary logic. Fuzzy logic can be applied in CR networks. In [18], fuzzy logic is used for the representation of cross-layer information and for the implementation of optimization strategies in CR networks. The fuzzy reasoning model that is appropriate for SU devices operating in heterogeneous networks is proposed in [19]. Fuzzy comprehensive evaluation is used for collaborative spectrum sensing in CR networks [8,20]. Fuzzy collaborative spectrum sensing improves the performance in terms of the probability of detection and false alarms. However, introducing fuzzy logic at node-level sensing can further improve the performance by improving local sensing results. In our proposed approach, fuzzy logic is used to make the local spectrum sensing decision.

\section{Spectrum sensing techniques}

The most commonly employed spectrum sensing techniques for transmitter detection are: matched filtering, energy detection, and cyclostationary detection. These spectrum sensing techniques are used for detection in parallel and then the fuzzy logic approach is used to determine spectrum holes. First, we will discuss each of the transmitter detection techniques including their pros and cons.
Recently, researchers have shown a great concern towards spectrum sensing to induce the effective interactivity of $\mathrm{CR}$ with the environment. One of the major spectrum sensing schemes is transmitter detection, in which we determine the frequency at which the transmitter is operating. A hypothesis model for transmitter detection is defined in [5] and models the signal received by the $\mathrm{SU}$ as

$$
r(t)=\left\{\begin{array}{cl}
n(t) & \text { when the PU is absent } \\
\mathbf{h} s(t)+n(t) & \text { when the PU is present }
\end{array}\right.
$$

where $r(t)$ is the signal received by SU, $s(t)$ is the signal transmitted by the PU, $n(t)$ is additive white Gaussian noise (AWGN), and $h$ is the amplitude gain of the channel. In general, the performance of spectrum sensing techniques is measured on the basis of two metrics: the probability of detection and the probability of false alarms. The probability of detection is the probability of SU's correctly declaring the presence of a PU and the probability of false alarms is defined as the probability of falsely declaring the presence of a PU. For the best performance, the probability of detection should be high and the probability of a false alarm should be low.

\subsection{Matched filter detection}

One of the well-known techniques in the field of signal processing for identifying a known pattern from a received signal is matched filter detection. In the presence of additive stochastic noise, the matched filter is an optimal linear filter for maximizing the SNR [21]. Figure 2 depicts the block diagram of a matched filter. The signal $r(t)$ received by SU is fed to the matched filter and is expressed mathematically as

$$
r(t)=\mathbf{h} s(t)+n(t)
$$

which is a generic form of $(1)$ and $s(t)$ is 0 if the $\mathrm{PU}$ is absent. The matched filter is equivalent to convolving the received signal $r(t)$ with a time-reversed version of the known signal or template as

$$
r(t) * s(T-t+\tau) .
$$

where $T$ is the symbol time duration and $\tau$ is the shift in the known signal.

Finally, the output of the matched filter is compared with a threshold factor $\lambda_{1}$ in order to decide whether the $\mathrm{PU}$ is present on the sensed spectrum.

The intuition behind the matched filter relies on the prior knowledge of the PU waveform such as 


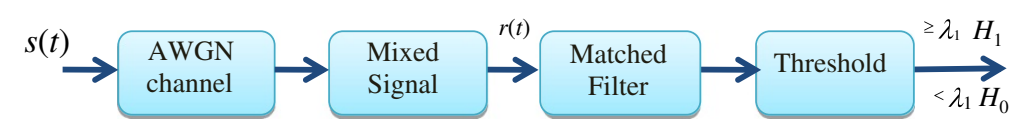

Figure 2 Block diagram of matched filter.

modulation type, order, the pulse shape, and the packet format. In order to meet such a stringent condition, CRs need to have a cache for pattern information in their memory and satisfy synchronization. Achieving synchronization is the most cumbersome part of demodulation. However, synchronization is still realizable because most PUs have pilots, preambles, synchronization words, or spreading codes that can be used for coherent detection [22].

The probability of detection, $P_{d, 1}$, and false alarm, $P_{f, 1}$, of a matched filter are given [23] as

$$
\begin{aligned}
& P_{d, 1}=Q\left(\frac{\lambda_{1}-E}{\sigma_{w} \sqrt{E}}\right) . \\
& P_{f, 1}=Q\left(\frac{\lambda_{1}}{\sigma_{w} \sqrt{E}}\right) .
\end{aligned}
$$

where $Q$ is the Gaussian complexity distribution function, $E$ is the energy of the deterministic signal of interest, and $\sigma_{w}^{2}$ is the noise variance.

\subsection{Energy detection}

When it is difficult for the SU to bring adequate information about the PU waveform, matched filter detection is not a favorable choice. However, if the SU is given the power of random Gaussian noise, energy detection becomes a better alternative [22] for spectrum sensing. Figure 3 depicts the block diagram for energy detection. The elementary approach behind energy detection is the estimation of the power of the received signal $r(t)$. To evaluate the power of the received signal, the output of a band pass filter of bandwidth $W$ is squared and integrated over an interval $T$. Finally, the integrated value is compared with a threshold $\lambda_{2}$ in order to decide whether the PU is present or not [24].
One of the major shortcomings of energy detection is that the performance is vulnerable to uncertainty in noise power. Energy detection determines the presence or absence of the PU based on the received signal energy. Since this detection scheme cannot discriminate between signal and noise power, it frequently causes false alarms at low SNR values [1].

The probability of detection, $P_{d, 2}$, and probability of false alarm, $P_{f, 2}$, of energy detection over the AWGN channel are approximated in [14] as

$$
\begin{aligned}
& P_{d, 2}=Q_{m}\left(\sqrt{2 \gamma}, \sqrt{\lambda_{2}}\right) . \\
& P_{f, 2}=\frac{\Gamma\left(M_{E}, \frac{\lambda_{2}}{2}\right)}{\Gamma\left(M_{E}\right)}
\end{aligned}
$$

where $\Gamma($.$) and \Gamma(.,$.$) are complete and incomplete gamma$ functions, respectively. $Q_{m}(.,$.$) is the generalized Marcum$ $Q$-function, $\gamma$ is the instantaneous SNR, $M_{E}$ is the time bandwidth product, and $\lambda_{2}$ is the decision threshold of the energy detector.

\subsection{Cyclostationary feature detection}

Researchers suggest that cyclostationary feature detection is more suitable than matched filter and energy detector techniques. As discussed earlier, the matched filter as a coherent detector requires prior knowledge about the PU's wave. Although the energy detector, as a non-coherent detection method, does not require any sort of prior knowledge about a PU's waveform and so is easy to implement, it is highly susceptible to in-band interference and changing noise levels [25] and cannot differentiate between signal and noise power.

Commonly the primary modulated waveforms are coupled with patterns also characterized as cyclostationary features like sine wave carriers, pulse trains, repeating spreading, hopping sequences, and cyclic prefixes

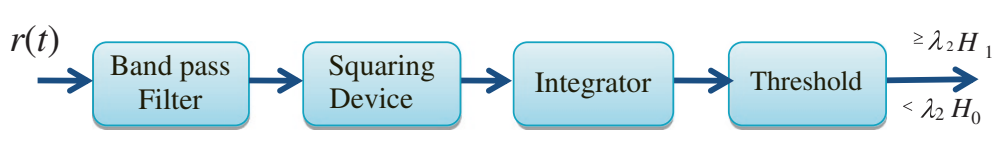

Figure 3 Block diagram of energy detection. 


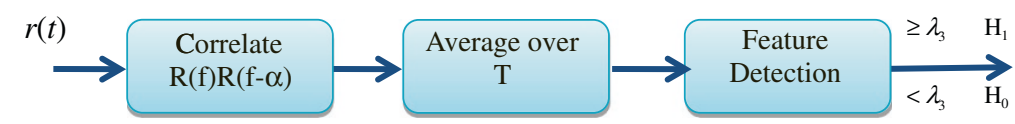

Figure 4 Block diagram of cyclostationary detection.

inducing periodicity [26]. SU can detect a random signal with a specific modulation type in the presence of random stochastic noise by exploiting periodic statistics like the mean and the autocorrelation of the PU waveform. Features like autocorrelation and mean are estimated by analyzing spectral correlation functions (SCFs). Implementation of the SCF is depicted in Figure 4.

SCF, also called a cyclic spectrum, is a two-dimensional function with a cyclic frequency $\alpha$. Power spectral density is a special case of a SCF with $\alpha=0$. The features detected are the number of signals, their modulation types, symbol rates, and presence of interferers. Using the computed SCF and a hypothesis model for spectrum sensing, we can determine whether a signal of a specific cyclic frequency of interest is present or not [27].

The probability of detection, $P_{d, 3}$, and probability of false alarm, $P_{f, 3}$, of one-order cyclostationary detection over an AWGN channel are approximated in [15] as

$$
\begin{aligned}
& P_{d, 3}=1-\left[1-Q_{m}\left(\frac{\sqrt{2 \gamma}}{\sigma_{m}}, \frac{\lambda_{3}}{\delta_{A}}\right)\right]^{L}, \\
& P_{f, 3}=1-\left(1-e^{-\frac{\lambda_{3}^{2}}{2 \delta_{A}^{2}}}\right)^{L} .
\end{aligned}
$$

where $\sigma_{w}^{2}$ is the variance, $\delta_{A}^{2}=\sigma_{m}^{2} /\left(2 M_{C}+1\right)$ in which $M_{c}$ is the number of samples for detection, $L$ is the number of diversity branches, $\gamma$ is instantaneous SNR, $Q_{m}(. .$. is the generalized Marcum $Q$-function, and $\lambda_{3}$ is a predetermined threshold.

\section{System model and framework}

Figure 5 illustrates a system model for the proposed spectrum sensing method. It is assumed that the PU signal structure is unknown but it allocates fraction of its power to transmit a deterministic pilot tone. This model is suitable for many practical communication systems in which the pilot tone is used for the data frame synchronization. A digital television (ATSC) signal is considered as the $\mathrm{PU}$ in which there is a strong pilot tone signal which is a sinusoidal signal in time domain. The spectrum sensing problem can be stated as where $f_{0}$ is the carrier frequency and $P_{s}$ and $\theta_{0}$ are the initial power and phase of the carrier, respectively. The signal $s(t)$ is modeled as $a(t) \cos \left(2 \pi f_{m} t\right)$ in which $f_{m}$ is the frequency of pilot tone in the TV signal and $a(t)$ is the analogue waveform. While on air, the signal becomes corrupted with AWGN noise $n(t)$. However, any interference from users operating in a nonoverlapping band can be mitigated through linear filtering. The SU will scan the whole spectrum and detect whether there is a spectrum hole available or not. All three transmitter detection techniques can be applied on this model and are compared in [23] using this model.

\subsection{FLD}

Traditional set theory has crisp concept of membership, i.e., an element either belongs to a set or it does not. In contrast, fuzzy set theory allows for partial membership. Fuzzy logic was initially proposed to cover the problem of reasoning under uncertainty. Decisions based on fuzzy logic are made using vague information, humanunderstandable fuzzy sets, and inference rules (e.g., IF, THEN, ELSE, AND, OR, and NOT) instead of complicated mathematics [8].

In order to test the applicability of fuzzy logic for the mathematical hypothesis given in (1) and increase the performance of local sensing, we propose an FLD scheme for the final decision (presence or absence of a PU). FLD offers several unique features that make itself a particularly good choice for PU detection. It does not require precise inputs therefore it is inherently robust. Because the FLD system is governed by user-defined rules, it can be modified easily to improve system performance. Figure 6 shows the structure of FLD system. When the input is applied to the FLD, the output is computed by the fuzzy inference engine corresponding to each rule. The crisp output is then computed by defuzzification from output sets. The system has three inputs and one output using singleton fuzzification, Max-Product, as the conclusion method and the center of area as the defuzzification method [7].

The FLD is designed to detect the PU accurately in order to increase reliability of the detection and to avoid

$$
r(t)=\left\{\begin{array}{cl}
n(t) & \text { when the PU is absent, } \\
2 \sqrt{P_{s}} s(t) \cos \left(2 \pi f_{0} t+\theta_{0}\right)+n(t) & \text { when the PU is present }
\end{array}\right.
$$




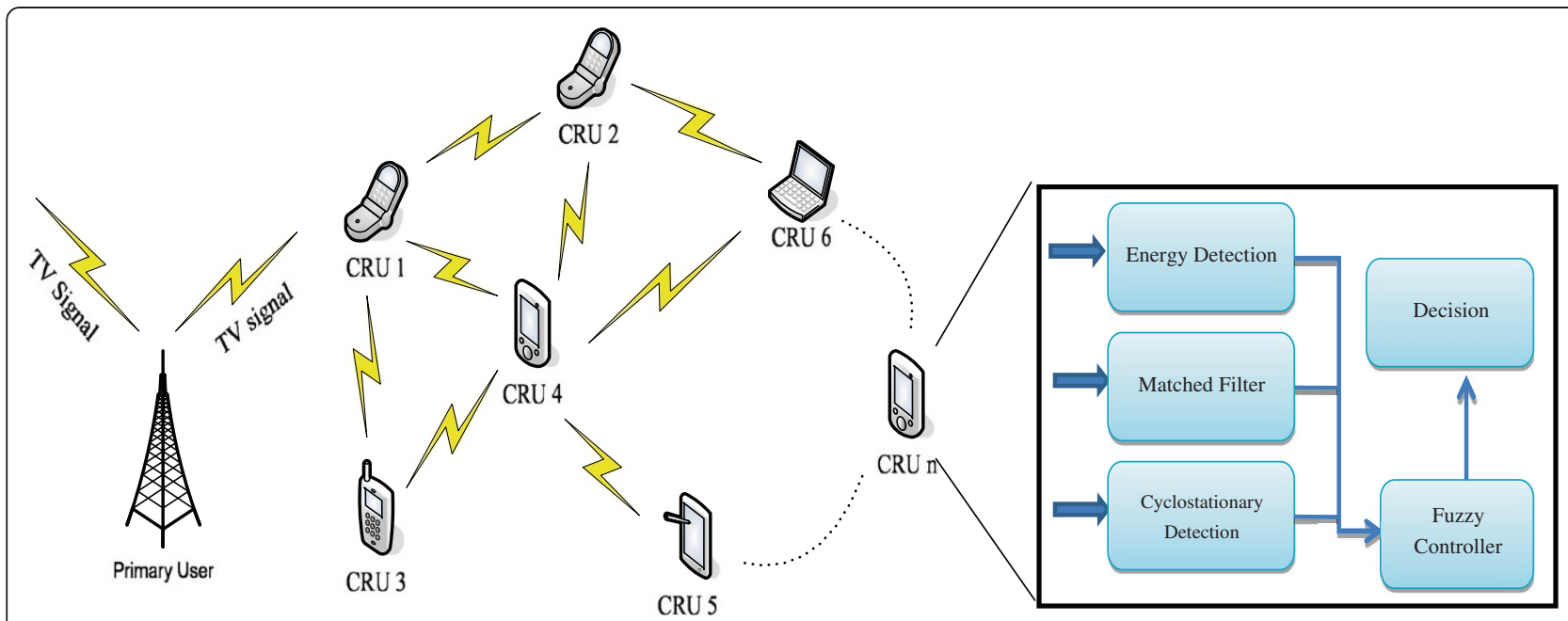

Figure 5 System model for fuzzy logic-based spectrum sensing.

interference with PU transmission. The detection of PU is based on three antecedents, i.e., descriptors

- Antecedent 1: Normalized output of energy detector

- Antecedent 2: Normalized output of matched filter

- Antecedent 3: Normalized output of cyclostationary detector

The linguistic variance used to represent each antecedent is labeled high, medium, or low, indicating the possibility of the presence of the PU. Each antecedent uses two thresholds for the label choice. Let $\lambda_{1 \mathrm{~A}}$ and $\lambda_{1 \mathrm{~B}}$ are two thresholds for which linguistic variance is high, medium, and low when the output of detector is greater than or equal to $\lambda_{1 \mathrm{~A}}$, between these two thresholds, and less than or equal to $\lambda_{1 \mathrm{~B}}$, respectively. In this article, $\lambda_{1 \mathrm{~A}}$ and $\lambda_{1 \mathrm{~B}}$ are set to be 0.25 and 0.75 , respectively. The consequence, i.e., the possibility of detection of $\mathrm{PU}$, is divided into seven levels which are worst, very bad, bad, moderate, good, very good, and best. The triangular membership function is used to represent high, medium, and low for all three antecedents as shown in Figure $7 \mathrm{a}$ and to represent worst, very bad, bad, moderate, good, very good, and best for the consequence as shown in
Figure $7 \mathrm{~b}$. Since there are 3 antecedents and 3 fuzzy subsets, there are totally 27 rules for the proposed FLD scheme. The fuzzy if-then rules in this FLD scheme are of these types:

$\mathrm{R}^{l}$ : IF $\mathrm{x}_{1}$ is $F_{l}^{1}$, and $\mathrm{x}_{2}$ is $F_{l}^{2}$, and $\mathrm{x}_{3}$ is $F_{l}^{3}$, THEN the possibility (y) that the PU is present is $\mathrm{D}^{l}$ where $l=1,2, \ldots$, 27.

Table 1 presents a rule base for combining the fuzzy information. The other rules can be interpreted in a similar way due to the symmetry of the rule base. The output $y$ from the FLD system is

$$
y\left(x_{1}, x_{2}, x_{3}\right)=\frac{\mu_{F_{l}^{1}}\left(x_{1}\right)+\mu_{F_{l}^{2}}\left(x_{2}\right)+\mu_{F_{l}^{3}}\left(x_{3}\right)}{N}
$$

where $N$ is the number of antecedents, in this case $N=3$, and $\mu_{F_{1}^{1}}, \mu_{F_{l}^{2}}$, and $\mu_{F_{l}^{3}}$ are the triangular membership functions for the all three antecedents. According to the draft IEEE 802.22 standard [27], the probability of false alarm and misdetection should be less than or equal to 0.1 . The available literature on setting a threshold value of individual detectors suggests that a target $P_{f}$ should be fixed to calculate required threshold. Therefore, the threshold $\lambda$ is set to 0.5 experimentally

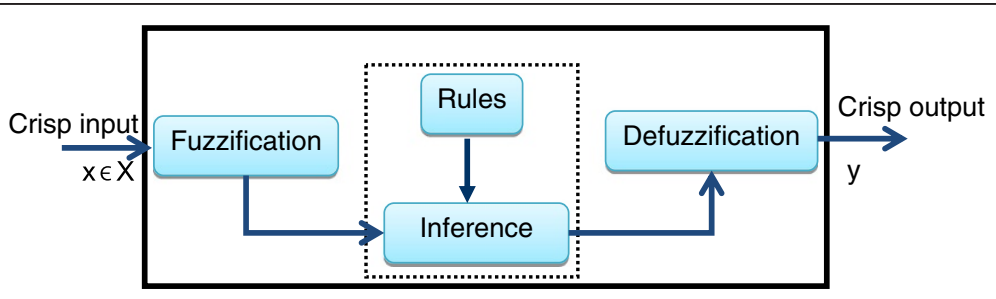

Figure 6 The structure of FLD system. 


\section{(a)}

(a)

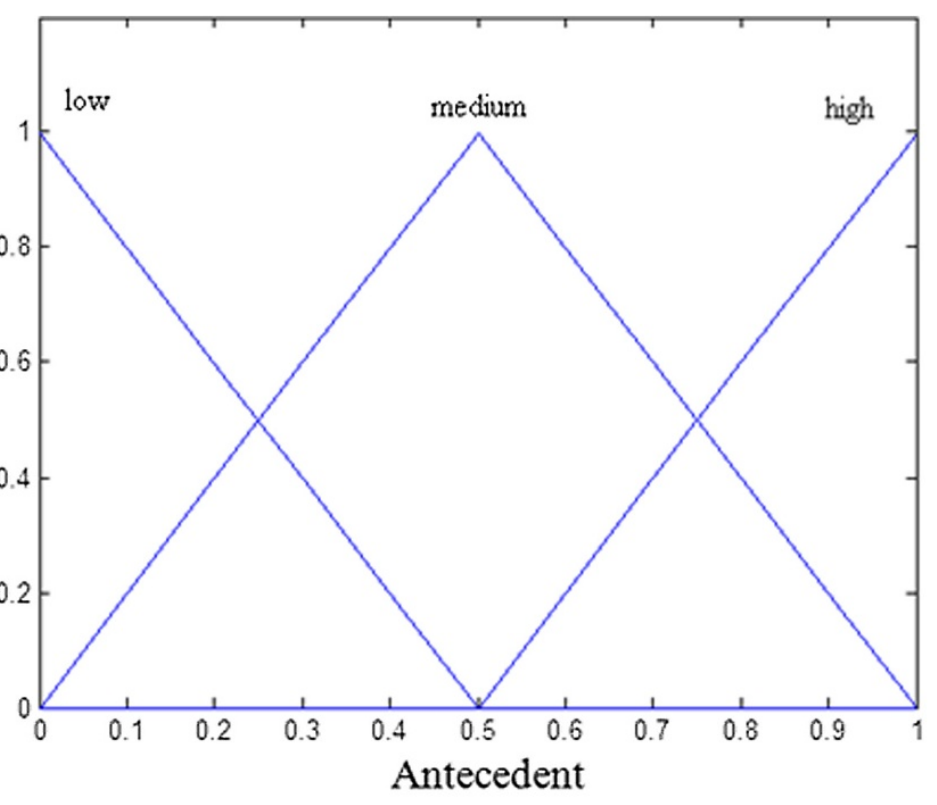

(b)

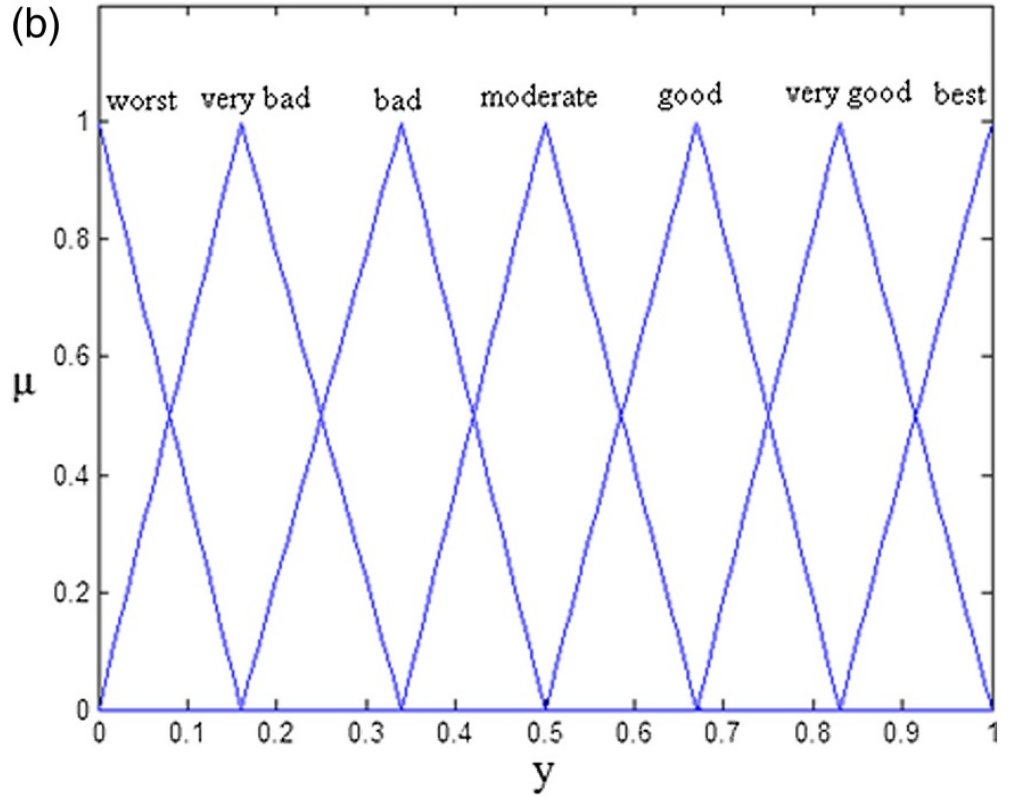

Figure 7 The membership functions used to represent the linguistic labels. (a) For all three inputs. (b) For the output.

to achieve $100 \%$ detection even for the probability of false alarm less than 0.1. If the decision metric $y$ is larger than the predetermined threshold, the $\mathrm{SU}$ will assert the presence of the PU. Otherwise, the SU will deny the presence of the PU.

\subsection{Analysis of sensing performance}

In this section, we analyze the sensing performance of proposed FLD scheme with respect to detection performance. The overall probability of detection and the probability of false alarm for the FLD scheme can be approximated as [28]

$$
\begin{aligned}
& P_{d}=\sum_{k=n}^{N} \sum_{\Sigma d_{i}=k} \prod_{i} P_{d, i}^{d_{i}}\left(1-P_{d, i}\right)^{1-d_{i}} \\
& P_{f}=\sum_{k=n}^{N} \sum_{\Sigma d_{i}=k} \prod_{i} P_{f, i}^{d_{i}}\left(1-P_{f, i}\right)^{1-d_{i}}
\end{aligned}
$$

where $d_{i}$ \{low, medium, high $\}$ for all $i=\{1, \ldots N\}$ and $n \geq N / 2 . P_{d, i}$ and $P_{f, i}$ are the probability of 
Table 1 Example of rule base for fuzzy combining

\begin{tabular}{llll}
\hline Input 1 & Input 2 & Input 3 & Output \\
\hline Low & Low & Low & Worst \\
Low & Medium & Low & Very bad \\
Low & Medium & Medium & Bad \\
Low & high & medium & Moderate \\
Medium & Medium & High & Good \\
High & Medium & High & Very good \\
High & High & High & Best \\
\hline
\end{tabular}

detection and probability of false alarm, respectively, when using $i$ th sensing technique. The summation based on $\sum d_{i}=k$ is used to include the effect of fuzzy logic and is conducted with all combinations of $d_{i}$ satisfying $\sum d_{i}=k$. It is shown in the next section that approximated probabilities are close to the simulation results.

In order to evaluate the agility of the FLD scheme, mean detection time is compared with matched filter detection, energy detection, and cyclostationary detection. The number of samples during observation periods is known in each sensing technique. The symbol duration is known in the case of the matched filter and the channel bandwidth is known for energy detection and cyclostationary detection. Using this information, we can calculate the mean detection time represented as $T_{1}, T_{2}$, and $T_{3}$ for matched filter, energy detection, and cyclostationary detection, respectively.
The mean sensing time for each channel for the matched filter, $T_{1}$, can be calculated as

$$
T_{1}=\frac{2 M_{1}}{T_{s}}
$$

where $M_{1}$ is the number of samples during the observation interval and $T_{s}$ is the symbol duration.

The mean sensing time for each channel for energy detection, $T_{2}$, can be calculated as

$$
T_{2}=\frac{M_{2}}{2 W}
$$

where $M_{2}$ is the number of samples during the observation interval and $W$ is the channel bandwidth.

The mean sensing time for each channel for cyclostationary detection, $T_{3}$, can be calculated as

$$
T_{3}=\frac{M_{3}}{2 W}
$$

where $M_{3}$ is the number of samples during the observation interval and $W$ is the channel bandwidth.

In the FLD scheme, all transmitter detection schemes run in parallel. The FLD will wait till all three detection algorithms finish their sensing. By doing so, the performance is increased and is better than individual performance of all the detectors. Main objective of this proposed FLD scheme is to increase reliability of detection at the cost of more hardware for each detector.

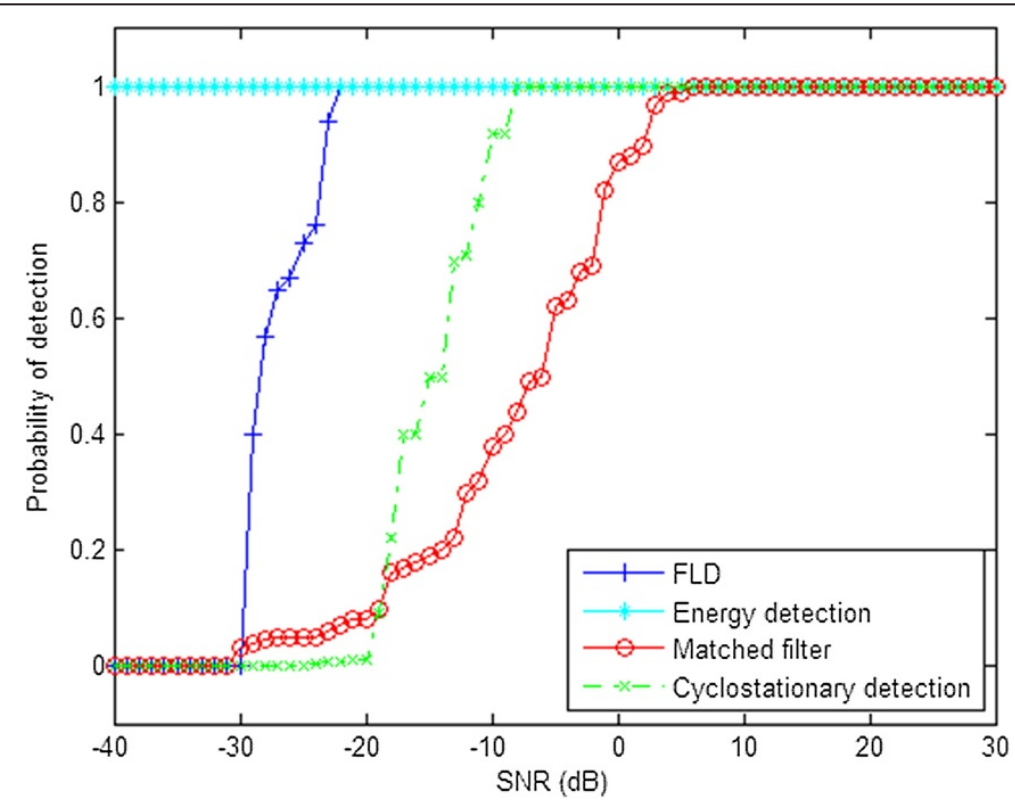

Figure 8 Comparison of transmitter detection and FLD schemes when the PU is present. 
Therefore, the total detection time of the proposed scheme can be expressed as

$$
\bar{T}=\operatorname{Max}\left(T_{1}, T_{2}, T_{3}\right)
$$

The probability of detection, $P_{d}$, and probability of false alarm, $P_{f}$, are calculated after combining the results of individual sensing techniques at each SU. Therefore, the overall $P_{d}$ is increased and $P_{f}$ is decreased when compared to individual techniques. Because the detection time of FLD scheme is equal to the maximum detection time of three sensing techniques, performance is improved with a similar sensing time at the cost of added parallel hardware at each SU.

\section{Simulation}

Figure 8 compares the probability of detection for transmission detection techniques with the proposed FLD scheme. The FLD scheme has a better performance over the entire SNR range compared to the other transmitter detection techniques. The FLD scheme detects the PU with $100 \%$ certainty even under a very low SNR value of $-22 \mathrm{~dB}$. In order to achieve the same degree of accuracy as a fuzzy logic scheme, the cyclostationary feature
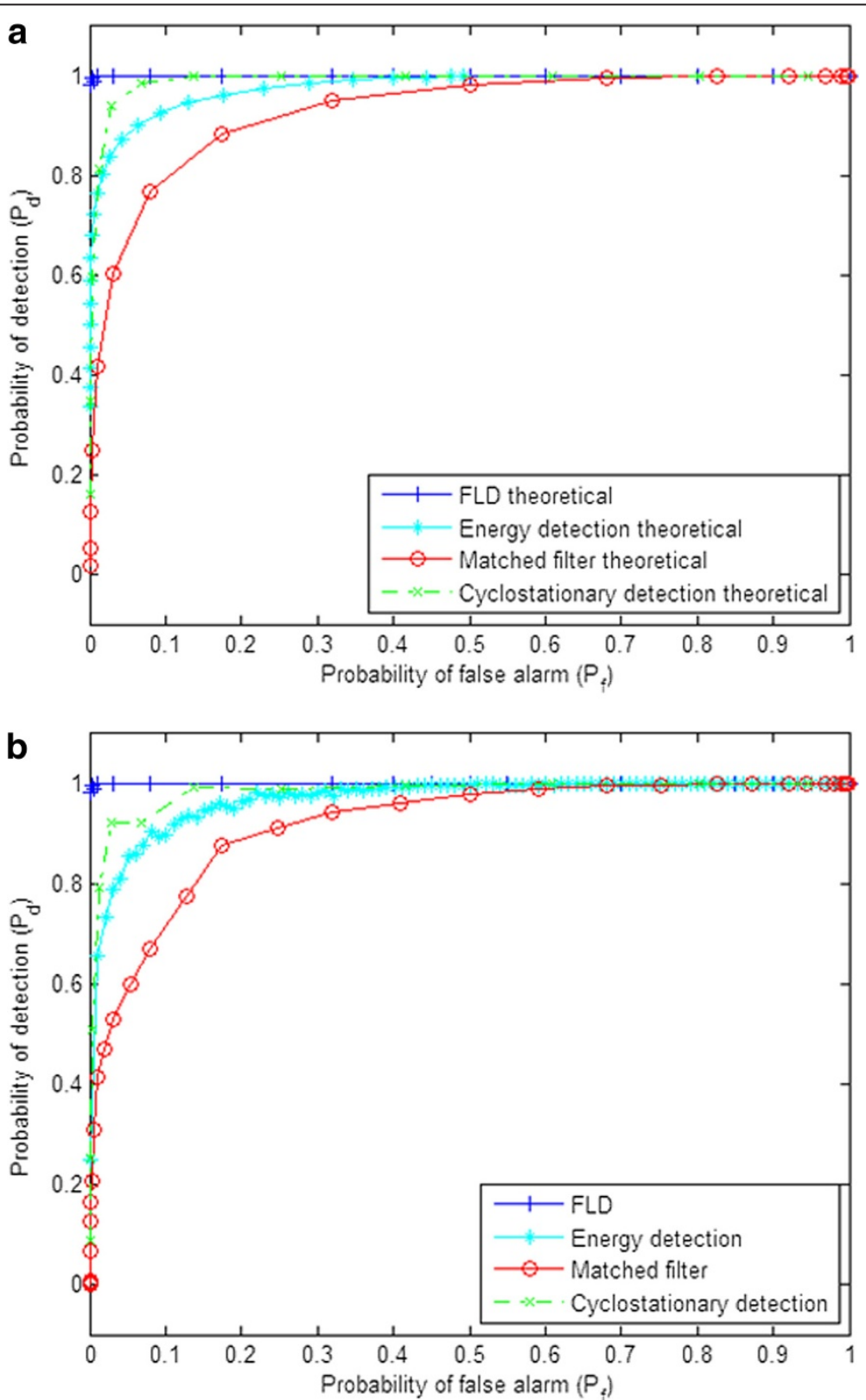

Figure 9 Comparison of transmitter detection and FLD schemes. (a) ROC theoretical. (b) ROC simulated (instantaneous SNR $=-10 \mathrm{~dB}$ ). 


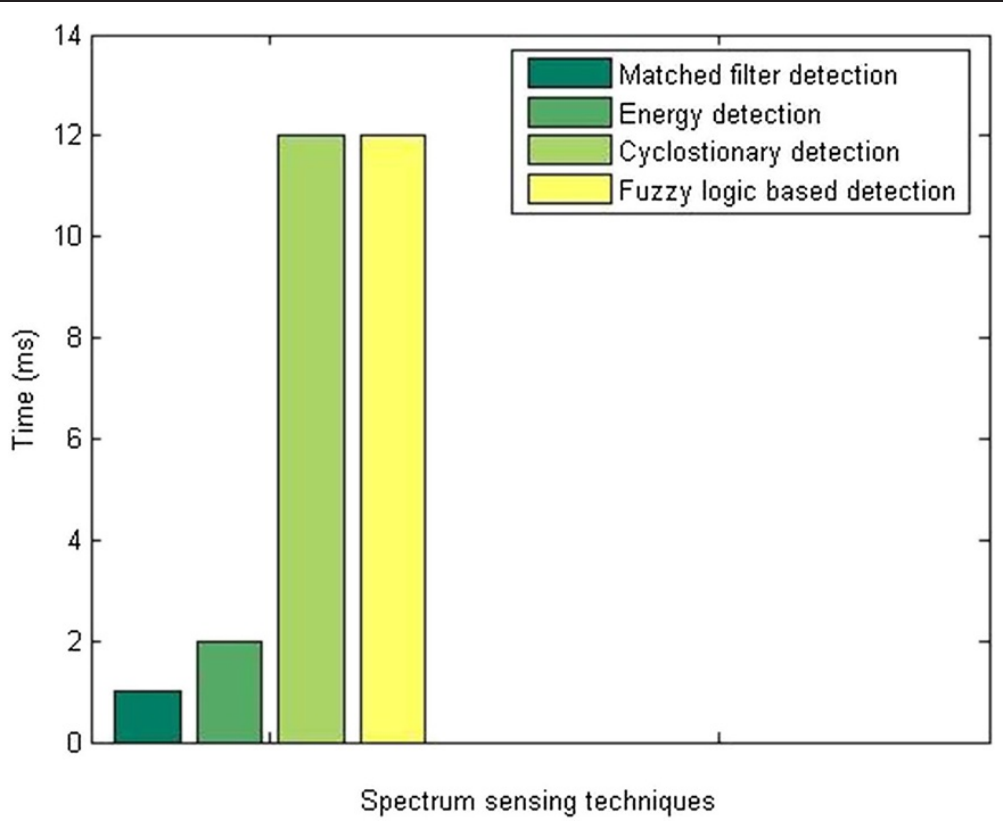

Figure 10 Sensing time of transmitter detection techniques compared with FLD.

detector and the matched filter require relatively higher SNR values of -8 and $2 \mathrm{~dB}$, respectively. The performance of energy detection seems to be better than all other mentioned techniques over the entire SNR range at the cost of high probability of false alarms. Due to inherent limitation of the energy detector, it is unable to discriminate between signal and noise energy. The probability of false alarm for the energy detection is the highest as compared to other detectors, which means that this comparison is not enough to determine the best detector for spectrum sensing.

In Figure 9, the receiver operating curves (ROCs) of the matched filter, energy detector, cyclostationary detector, and FLD scheme are shown. In this scenario, it is assumed that the instantaneous SNR is $-10 \mathrm{~dB}$. Time bandwidth product $M_{E}$ for energy detection is 2. Noise variance $\sigma_{w}^{2}$ for both matched filter and cyclostationary detection is taken as $1 \mathrm{~dB}$. The number of samples for detection $M_{c}$ and the number of diversity branches $L$ for cyclostationary detection are assumed to be 2 and 3 , respectively. It is seen that the simulated ROCs shown in Figure $9 \mathrm{~b}$ agree with the theoretical results presented in Figure 9a. The result shows that the ROC performance of FLD scheme outclasses all the existing transmitter detection techniques. FLD scheme is an optimal choice even at lower SNR values.

Figure 10 shows the comparison of the detection time of the proposed FLD scheme with matched filter, energy

Table 2 Comparison of the proposed scheme with existing improved local sensing techniques

\begin{tabular}{|c|c|c|c|}
\hline Spectrum sensing scheme & Average SNR (dB) & $P_{f}$ & $P_{d}$ \\
\hline \multirow[t]{2}{*}{ Proposed FLD scheme } & -15 & 0.0001 & 0.50 \\
\hline & -10 & 0.0001 & 0.97 \\
\hline \multirow[t]{2}{*}{ PU detection in a multiple antenna CR [9] } & 0 & 0.01 & 0.45 \\
\hline & 15 & 0.01 & 1 \\
\hline A two-stage sensing technique for dynamic spectrum access [12] & 6 & 0.1 & 0.99 \\
\hline Two-stage spectrum sensing for CRs [13] & -10 & 0.1 & 0.99 \\
\hline Combined energy detection and one-order cyclostationary detection [14] & 10 & 0.1 & 0.94 \\
\hline Energy detector with bi-thresholds [15] & 10 & 0.1 & 0.85 \\
\hline \multirow[t]{2}{*}{ Improved energy detector [16] } & 0 & 0.1 & 0.62 \\
\hline & 10 & 0.1 & 0.99 \\
\hline \multirow[t]{2}{*}{ Advanced sensing techniques of energy detection [17] } & -5 & 0.1 & 0.72 \\
\hline & -5 & 0.6 & 0.95 \\
\hline
\end{tabular}


detection, and cyclostationary detection. In proposed FLD scheme, all spectrum sensing techniques run in parallel and so detection time is the maximum time taken by any individual detection scheme, while detection performance comparatively increases relative to any individual detection performance. The key advantage of the proposed FLD scheme is that its detection performance is more reliable than existing spectrum sensing techniques with a mean sensing time equal to that of cyclostationary detection.

In Table 2, we compared the performance of proposed FLD scheme with existing, improved local sensing techniques as discussed in Section 2. The two-stage spectrum sensing proposed in [13] gives the most reliable results in comparison with the remaining existing schemes mentioned in Table 2 . The probability of detection of the proposed FLD scheme is 0.97 in comparison with 0.99 for the two-stage spectrum sensing scheme, at an average SNR of $-10 \mathrm{~dB}$. However, FLD has a false alarm probability of 0.0001 , while the corresponding value for the two-stage spectrum sensing scheme is 0.1 . False alarms reduce spectral efficiency and misdetection causes interference with the PU. In general, it is then vital for optimal detection performance that the maximum probability of detection is achieved with the minimum probability of false alarm [6]. Therefore, the advantage of the FLD scheme is that it achieves a high probability of detection with a minimum probability of false alarms in comparison with existing schemes at low SNR values.

\section{Conclusion}

In this article, a new FLD scheme for local spectrum sensing is proposed. In the first stage of FLD, each SU performs existing spectrum sensing techniques, i.e., energy detection, matched filter detection, and cyclostationary detection, in parallel. In the second stage, the outputs of those detection approaches are combined using fuzzy logic in order to deduce the presence or absence of PU.

Transmitter detection techniques are compared with the proposed fuzzy logic-based approach. By comparing these techniques, we conclude that the FLD scheme gives better results in terms of the probability of detection and false alarms. The FLD scheme has a mean detection time equal to the maximum time taken by any existing scheme, i.e., the mean detection time of cyclostationary detection. All the existing techniques perform at each SU in parallel, and therefore the hardware cost of the proposed FLD is slightly higher. However, since accurate detection is to be predicted, cost can be sacrificed for the accuracy of detection and fast detection time.

Every detection technique has an SNR threshold below which robust operation is not possible. We find that by simultaneously combining the results of different detection techniques using fuzzy logic, better results can be obtained.

\section{Competing interests}

The authors declare that they have no competing interests.

\section{Acknowledgement}

This work was supported by the CITRC (Convergence Information Technology Research Center) support program (NIPA-2012-H0401-12-1003) supervised by the NIPA (National IT Industry Promotion Agency) of the MKE. It was partially supported by Seoul R\&BD Program (SS110012C0214831) and Special Disaster Emergency R\&D Program from National Emergency Management Agency (2012-NEMA10-002-01010001-2012).

\section{Author details}

'Department of Information and Communication Engineering, Sejong University, Seoul, Republic of Korea. ${ }^{2}$ School of Engineering and Information Sciences, Middlesex University, London, UK.

Received: 13 February 2012 Accepted: 8 October 2012 Published: 21 November 2012

\section{References}

1. I.F. Akyildiz, W.-Y. Lee, M.C. Vuran, M. Shantidev, Next generation / dynamic spectrum access / cognitive radio wireless networks: a survey. Comput. Netw. 50, 2127-2159 (2006)

2. S. Haykin, Cognitive radio: brain-empowered wireless communications. IEEE J. Sel. Areas Commun. 23(2), 201-220 (2005)

3. J. Ma, G. Li, B.H. Juang, Signal processing in cognitive radio. Proc. IEEE 97(5), 805-823 (2009)

4. A. Ghasemi, E.S. Sousa, Spectrum sensing in cognitive radio networks: requirements. Challenges and Design Trade-offs. IEEE Commun. Mag. 46(4), 32-39 (2008)

5. T. Yucek, H. Arslan, A survey of spectrum sensing algorithms for cognitive radio applications. IEEE Commun. Surv. Tutor. 11(1), 116-130 (2009)

6. I.F. Aklidz, B.F. Lo, R. Balakrishan, Cooperative spectrum sensing in cognitive radio networks: a survey. Phys. Commun. 4(1), 40-62 (2011)

7. M. Matinmikko, T. Rauma, M. Mustonen, I. Harjula, H. Sarvanko, A. Mammela, Application of fuzzy logic to cognitive radio systems. IEICE Trans. Commun. E92-B(12), 3572-3580 (2009)

8. T.K. Xuan, I. Koo, A. Cooperative, Spectrum sensing scheme using fuzzy logic for cognitive radio networks. KSII Trans. Internet Inf. Syst. 4(3), 289-304 (2010)

9. A. Pandharipande, J.-P.M.G. Linnartz, Performance analysis of primary user detection in a multiple antenna cognitive radio. Proc. IEEE Conf. Commun (Glasgow, 2007), pp. 6482-6496. June 2007

10. D.S. Shiu, G. Foschini, M. Gans, J. Kahn, Fading coorelation and its effect on the capacity of multielememt antenna systems. IEEE Trans. Commun. 48(3), 502-513 (2000)

11. Y. Hur, J. Park, W. Woo, K. Lim, C.-H. Lee, H.S. Kim, J. Kaskar, A Widband Analog Multi-Resolution Spectrum Sensing (MRSS) Technique for Cognitive Radio (CR) Systems. IEEE International Symposium on Circuits and Systems (Kos, 2006), pp. 4090-4093. May 2006

12. L. Luo, N.M. Neihart, S. Roy, D.J. Allstot, A two-stage sensing technique for dynamic spectrum access. IEEE Trans. Wirel. Commun. 8(6), 3028-3037 (2009)

13. S. Maleki, A. Pandharipande, G. Leus, Two-stage spectrum sensing for Cognitive Radios. IEEE International Conference on Acoustic Speech and Signal Processing (Dallas, 2010), pp. 2946-2949. May 2010

14. W. Yue, B. Zheng, Q. Meng, W. Yue, Combined energy detection and oneorder cyclostationary feature detection techniques in cognitive radio systems. J. China Univ. Posts Telecommun. 17(4), 18-25 (2010)

15. W. Yue, B. Zheng, Spectrum sensing algorithms for primary detection based on reliability in cognitive radio systems. J. Comput. Electr. Eng. 36(3), 469-479 (2010)

16. Y. Chen, Improved Energy Detector for Random Signals in Gaussian Noise. IEEE Trans. Wirel. Commun. 9(2), 558-563 (2010)

17. H. Wang, G. Noh, D. Kim, S. Kim, D. Hong, Advanced Sensing Techniques of Energy Detection in Cognitive Radios. J. Commun. Netw. 12(1), 19-29 (2010)

18. N. Baldo, M. Zorzi, Fuzzy Logic for cross-layer optimization in cognitive radio networks. IEEE Commun. Mag. 46(4), 64-71 (2008) 
19. A. Merentitis, E. Patouni, N. Alonistioti, M. Doubrava, To reconfigure or not to reconfigure: Cognitive mechanisms for mobile devices decision making. IEEE 68th Vehicular Technology Conference (Calgary, 2008), pp. 1-5. Sep. 2008

20. Y. Wendong, Y. Yuhuang, C. Yueming, X. Youyun, A collaborative spectrum sensing scheme using fuzzy comprehensive evaluation in cognitive radio. J. Electron. (China) 26(3), 326-331 (2009)

21. B. Saklar, Digital Communications: Fundamentals and Applications, 2nd edn. (Prentice Hall, Upper Saddle River, 2001)

22. D. Cabric, A. Tkachenko, R.W. Brodersen, Spectrum sensing measurements of pilot, energy, and collaborative detection. IEEE Military Comm Conference (Washington, 2007), pp. 1-7. Oct. 2007

23. D. Bhargavi, C.R. Murthy, Performance Comparison of Energy, Matched-Filter and Cyclostationarity-Based Spectrum Sensing. IEEE Eleventh International Workshop on Signal Processing Advances in Wireless Communications (Morocco, 2010), pp. 1-5. Dec. 2010

24. R. Tandra, A. Sahai, SNR walls for signal detection. IEEE J. Sel. Topics Signal Process. 2(1), 4-17 (2008)

25. A. Fehske, J. Gaeddert, J.H. Reed, A New Approach to Signal Classification Using Spectral Correlation and Neural Networks. First IEEE Symposium on New Frontiers in Dynamic Spectrum Access Networks (Baltimore, 2005), pp. 144-150. Nov. 2005

26. D. Cabric, S.M. Mishra, R.W. Brodersen, Implementation Issues in Spectrum Sensing for Cognitive Radios. Proceedings of the 38th Asilomar Conference on Signals Systems and Computers (Pacific Grove, 2004), pp. 772-776. Nov. 2004

27. S.-Y. Chang, Analysis of Proposed Sensing Schemes. IEEE P802.22-06/0032r0 Wireless RANs (2006)

28. L. Ling, L. Yin, Z. Hongbo, Half-Voting Based Twice-Cooperative Spectrum Sensing in Cognitive Radio Networks. International Conference on Wireless Communications, Networking and Mobile Computing (Beijing, 2009), pp. 1-3. Sep. 2009

doi:10.1186/1687-6180-2012-242

Cite this article as: Ejaz et al:: Improved local spectrum sensing for cognitive radio networks. EURASIP Journal on Advances in Signal Processing 2012 2012:242

\section{Submit your manuscript to a SpringerOpen ${ }^{\circ}$ journal and benefit from:}

- Convenient online submission

- Rigorous peer review

- Immediate publication on acceptance

- Open access: articles freely available online

- High visibility within the field

- Retaining the copyright to your article

Submit your next manuscript at $\gg$ springeropen.com 